CUNEO M.M., «Decisiones, emociones y bioética. Apuntes neurocientíficos» en Moralia 38, (2015) 179-191. También publicado (con autorización del director de la revista Moralia) en Stromata 71, (2015)13-25.

\title{
Decisiones, emociones y bioética Aportes neurocientíficos
}

\author{
María Martha Cúneo h.m.r. ${ }^{1}$
}

\section{Cuando decidir no es fácil...}

Vivimos decidiendo. Desde decisiones pequeñas y de mínima complejidad a aquellas que implican toda nuestra vida. Existen incluso decisiones en las cuales ponemos en juego lo esencial de nuestra existencia, como son las decisiones de vida o muerte. Decisiones extremas son esas decisiones que uno tiene que tomar en la urgencia y no hay posibilidad de poder esperar a que la situación esté básicamente estabilizada o calmada. En nuestro quehacer cotidiano nos encontramos con situaciones que nos enfrentan a este tipo de decisiones. Jugarse o no por una persona o una situación a riesgo de nuestra integridad personal, económica o social, decisiones que tocan nuestros dolores más profundos y que nos obligan a re-acomodarlos.

Uno de los campos en el que los agentes morales se ven frecuentemente enfrentados con este tipo de decisiones, es el campo de la salud. Los profesionales de la salud se enfrentan a situaciones que exigen decisiones extremadamente delicadas que comprometen y disparan todos sus móviles interiores. Exigen y muestran todas las dimensiones de su persona, en acto. Muchas veces son bien manejadas. A pesar de ser decisiones que conllevan un porcentaje de incertidumbre y stress, hay profesionales que las enfrentan y las resuelven sin grandes problemas. En cambio hay otros profesionales que son superados por estas situaciones. Y ese desborde se expresa a través de diferentes reacciones. Desde la negación o el escape al síndrome del burnt out.

Pensar y decidir éticamente, aun en situaciones difíciles, también se entrena. Fue la base de toda ascesis en la moral tradicional a través de las virtudes. Incluso en la moral tradicional se percibió que las decisiones no solo eran una cuestión de razones. ¿Es acaso el componente racional lo más medular de nuestras decisiones? ¿Cómo se relacionan lo racional y lo emocional? ¿Cómo interaccionan estos elementos en una conciencia que decide imperada por la urgencia en una situación clínica límite?

Re-pensar una formación y un entrenamiento ético, en el campo de los agentes de salud, es lo que nos ha llevado a preguntarnos por el status questionis de la naturaleza de los juicios morales al integrar los nuevos aportes de la neurociencia y la psicología moral e intentar ver cómo cooperar para delinear una formación consciente orientada a abordar este tipo de decisiones lo mejor posible.

\section{Nuestra toma de decisiones de cada día}

\footnotetext{
${ }^{1}$ Hermana Misionera Redentorista. Médica (Universidad de Buenos Aires), doctora en Teología Moral (Academia Alfonsiana), profesora de Teología Moral en la Facultad de Teología de la Universidad Católica Argentina y de la Facultad de Teología de la Universidad del Salvador. Miembro del Comité de Bioética del Hospital de Niños de Buenos Aires, Dr. Ricardo Gutiérrez y de la Sub-Comisión de Ética de la Sociedad Argentina de Pediatría. mmcuneohmr@yahoo.com.ar
} 


\section{¿Cómo decidimos y qué decidimos?}

Dentro de las decisiones morales debemos considerar dos cosas. Una es el proceso o estructura decisional (decision making) que correspondería a la pregunta acerca de "cómo decidimos". Otra cosa es "qué decidimos", apuntando al contenido de las decisiones: esta segunda dimensión se relaciona con la pregunta ¿qué es lo bueno? A ella se han referido por ejemplo los sistemas morales y las diferentes teorías y paradigmas que hoy se utilizan para fundamentar la moral. Nos focalizaremos en el primer punto, especialmente en la secuencia que se activa en el proceso de decisión, aunque las estructuras que se activan no dejan de ser precisamente indicativas del contenido que se terminará eligiendo.

La conciencia fue clásicamente concebida como el lugar de las decisiones. La escolástica contó con distintas interpretaciones acerca del esquema básico de la conciencia habitual y actual ${ }^{2}$, dada por las escuelas medievales tomista, franciscana y mística. Para la primera, el proceso de decisión de la conciencia, era un pulcro silogismo de razón en un limpio y claro pensamiento deductivo. La escuela franciscana suaviza este racionalismo y vincula el proceso de decisión de la conciencia con la voluntad que busca el bien. La escuela mística, en cambio, descarta que sea el intelecto o la voluntad la que se mueva hacia el bien, sino que el motor procesual es la experiencia cercana de Dios.

A la conciencia se la interpretó principalmente como razón que actúa, hasta mediados del siglo pasado. A partir de allí, además de señalarse un marcado componente religioso, se apuntó a una concepción mucho más global. De esta manera, la reflexión sobre la conciencia se abrió a otras vertientes humanas, caminó hacia una mayor afinidad con la idea bíblica de corazón y permitió la posibilidad de integrar los nuevos datos de la moderna psicología. Precisamente fue la psicología moral, en la vertiente de Piaget y Kohlberg, la que en la década del 60-70 intentó delimitar mejor los componentes claves de la estructura y proceso de la decisión ${ }^{3}$. La línea de estos autores fue netamente racionalista neokantiana ${ }^{4}$. La teoría fue elaborada en base a los conceptos de bien, deber y justicia ${ }^{5}$. El posterior aporte de Carol Guilligan no solo consistió en mostrar la "voz diferente" de la forma de decidir femenina, sino que el planteo de Carol puso en evidencia un elemento que va más allá de las fronteras marcadas por la diferencia de género. Es el elemento de la inclusión de lo afectivo y lo emocional en el juicio moral, elemento que Kolberg no consideró suficientemente, ya que partía de bases racionalistas. La psicología cognitiva post-kohlbergiana ayudó a la Teología Moral a entender mejor la influencia del factor afectivo en el juicio moral y la toma de

\footnotetext{
${ }^{2}$ Cf.: WeBer H., Teología Moral General. Exigencias y respuestas, Herder, Barcelona 1994, 251-275.

${ }^{3}$ Cf.: Weber H., Teología Moral General. Exigencias y respuestas, Herder, Barcelona 1994, 316-329.

${ }^{4}$ «todo el mundo ha observado el paralelismo que existe entre las normas morales y las normas lógicas: la lógica es una moral del pensamiento como la moral es una lógica de la acción», PIAGET J., El criterio moral en el niño, Barcelona, $1977^{3}$, 335. La moral consiste en un sistema de reglas y la esencia de cualquier moral hay que buscarla en el respeto que el individuo adquiere hacia estas reglas. El análisis reflexivo de Kant, la sociología de Durkheim o la psicología individualista de un Bovet, coinciden en este punto. Las divengencias doctrinales aparecen solo cuando se trata de explicar cómo llega la conciencia al respeto por estas reglas. Ibid. 9. «El juicio moral, como expresión racional de la moralidad pasa por dos etapas: de la mera aceptación de la norma (Durkheim) a una cooperación basada en el respeto (Bovet)». MIFSUD T., «Los diversos estadios del desarrollo moral», en YÁÑEZ H. M. (E.D.), Desarrollo moral y educación afectiva, San Benito, 50.
}

${ }^{5}$ Cf.: Mifsud T., «Los diversos estadios del desarrollo moral», en YÁÑEZ H. M. (E.D.), Desarrollo moral y educación afectiva, San Benito, 51. 
decisión ética ${ }^{6}$. «Los sentimientos y las emociones impregnan los actos del sujeto moral, la sensibilidad moral, el temperamento, la personalidad moral etc. De esta manera, una decisión moral no es solamente un proceso racional, sino también emotivo» ${ }^{7}$.

Actualmente, la filosofía moral, impulsada especialmente desde el ámbito de la neuroética, ha aportado elementos acerca del funcionamiento de nuestro cerebro y el juego de las emociones en la formación del juicio moral, intentando progresar con este dato en el conocimiento del fenómeno moral ${ }^{8}$.

\section{3. ¿Decidimos en conciencia? Neurobiología de la toma de decisiones}

El lugar de lo emotivo en nuestras decisiones fue abordado primeramente por la psicología moral ${ }^{9}$. Ha sido el psicólogo social Jonathan Hait quién en su artículo titulado «El perro emocional y su cola racional» ${ }^{10}$, afirmó que si bien fue valioso el aporte de los autores de la posición racional como respuesta a la pregunta acerca de cómo formulamos los juicios morales, eso no es ciento. Para Hait hacemos nuestros juicios morales intuitivamente, o sea de forma inmediata y automática. Ante dilemas morales no razonamos y no sabemos si seguimos un proceso. Siempre se activa el componente emocional, que reacciona intuitiva y rápidamente. Este autor presenta una serie de dilemas en que la gente termina diciendo "no se por qué, pero sí se que está mal". El razonamiento viene después, justificando el punto de partida que rara vez se cambia. Con el razonamiento no se busca la verdad, sino tan solo justificar lo realizado. Decidimos emocionalmente y luego damos razones. La pregunta que esto suscita es: ¿de dónde vienen esos juicios que se tomaron sin argumentos racionales? ¿Ya los tenemos escritos en el cerebro? ¿Habría un "cableado" previo que pueda justificarlo?

Posteriormente fueron los artículos de Joshua Greene, neurocientífico de Harvard, quién por medio de la resonancia magnética por imágenes, puso la atención en las zonas del cerebro que se activan a la hora de presentarse diversos dilemas morales.

\footnotetext{
${ }^{6}$ Cf: BoIs M., «Hacia una comprensión psico-teológico-moral del juicio moral», Moralia 31 (2008) 39-64.

${ }^{7}$ Ibid. 41.

${ }^{8}$ Cf.: PÉREZ Zafrilla, P., «Implicaciones normativas de la psicología moral: Jonathan Haidt y el desconcierto moral», en Daímon, 59, (2013), 9-25. CoRTINA A., «Neuroética: ¿las bases cerebrales de una ética universal con relevancia política?», en Isegoría, 42, (2010), 129-148; 132.

${ }^{9}$ Cf.: CORTINA A., «Neuroética: ¿Ética fundamental o ética aplicada?», en LÓPEZ FríAs, F., Y OTROS (EDS.) Bioética, neuroética, libertad y justicia, Ediciones Comares, Granada 2013, 802-830. CorTINA A., Neuroética, ¿moral como estructura o como contenido?, Conferencia dictada $1^{\circ}$ de septiembre de 2010 en el marco del III $^{\circ}$ Congreso Internacional Xavier Zubiri, Pontificia Universidad Católica de Valparaíso, Chile, en http://www.youtube.com/watch?v=QzM7 dKGMSs . (Consultado el 12.9. 2013).

${ }^{10}$ HAIDT J., «The emotional dog and its rational tail. A social Intuitionist approach to moral judgement», en Psychological Review, 108/4, 2001, 814-834. Aunque es importante decir que el autor no habla de emoción como pasión contraria a razón, sino como intuición, como una dimensión cognitiva valida, como la razón. «Esta dimensión cognitiva de la emoción es un punto no subrayado de manera suficiente por Haidt en sus primeros trabajos sobre intuicionismo social (como tampoco harán sus críticos). Esto es algo que él mismo lamentará en su última obra, The righteous mind. En ella, lamenta haber titulado su famoso trabajo de 2001 como «El perro emocional y su cola racional» y no como «El perro intuitivo...» ya que el primer título induce a pensar que las emociones carecen de valor cognitivo al oponer lo emocional a lo racional, según se entiende desde el paradigma tradicional racionalista», en PÉREZ ZAFRILLA, P., «Implicaciones normativas de la psicología moral: Jonathan Haidt y el desconcierto moral», Daímon, 59 (2013), 9-25; 11-12.
} 
"Como es sabido, las neurociencias son ciencias experimentales que tratan de explicar cómo funciona el cerebro, sobre todo el humano, valiéndose del método de observación, experimentación e hipótesis, propio de las ciencias empíricas, y de las herramientas técnicas disponibles. Dieron un paso prodigioso al descubrir que las distintas áreas del cerebro se han especializado en diversas funciones y que a la vez existe entre ellas un vínculo. Las técnicas de neuroimagen, tanto la resonancia magnética estructural como la funcional, permiten descubrir no sólo la localización de distintas actividades del cerebro, sino también las actividades mismas, el "cerebro en acción", y son estas técnicas las que han promovido un extraordinario avance en las neurociencias" 11 .

Greene $^{12}$ observó que las diferentes áreas cerebrales se activaban frente a dilemas de dos tipos: personales e impersonales. Se percibió que cuando los dilemas fueron personales, o sea, referidos personajes del mundo cotidiano, el cerebro respondía desde las zonas emotivas e inmediatamente optaba por una respuesta ética. En cambio no sucedía lo mismo con aquellos dilemas que se referían a personas lejanas, expresadas a través de sustantivos colectivos. El cerebro tardaba más y los códigos utilizados eran racionales. Nos cuesta mucho esfuerzo racional decir que no hay que ayudar a una persona que vemos y que está a nuestro lado. La conclusión a la que se llega es que nos afectan mucho más los cercanos que los lejanos. Lo lejano no nos interesa. "Ojos que no ven, corazón que no siente", dice el refrán.

Wilson ${ }^{13}$, neurocientífico, intenta explicar posteriormente este fenómeno. En la época de formación del cerebro, el ser humano debía ayudar al propio grupo y defenderse de los lejanos en orden a su supervivencia.

Finalmente, lo que ha quedado en evidencia, es que los juicios morales vienen mediados en muy buena medida por la emoción. En la educación moral hemos tenido muy en cuenta el nivel del razonamiento pero no hemos tenido en cuenta el aprendizaje emocional. Las emociones son fundamentales en el terreno de la moral. Y se agregan datos importantísimos: el primero es que las emociones pueden cultivarse. El segundo es que las emociones tienen una enorme capacidad cognitiva ${ }^{14}$. Nos abren a un gran mundo; quien no tiene capacidad de indignación tampoco tendrá sentido de la justicia. Quien no tiene capacidad de conmoverse tampoco será capaz de percibir el sufrimiento de otros. El cultivo de las emociones en el terreno de la moralidad es fundamental ya que juegan un papel tan importante en las decisiones.

Los aportes de la neurociencia y la posibilidad de la visualización de las áreas del cerebro que usamos para decidir, nos exigen re-pensar los conceptos tradicionales acerca de los componentes del juicio moral. Todos los seres humanos nos basamos en nuestro aprendizaje, experiencia, intuición y emoción e integramos la información del contexto que cambia permanentemente de manera inmediata y automática ${ }^{15}$. Según investigaciones neurocientíficas, el área órbitofrontal es vital para el proceso de toma de

\footnotetext{
${ }^{11}$ CORTINA A., «Neuroética: ¿Ética fundamental o ética aplicada?», LOPEZ FrIAS, F., Y OTROS (EDS.) Bioética, neuroética, libertad y justicia, Ediciones Comares, Granada 2013, 804.

${ }^{12}$ Cf: GREENE J., ET ALT, «An fMRI Investigation of Emocional Engegement in Moral Judgement», Science 293, (2003) 2105-2108.

${ }^{13}$ Cf: WiLson D. S., «Group selection», en PAGEL M., (Comp.), Oxford Encyclopedia of Evolution, Oxford University Press, Oxford 2002, 450-454.

${ }^{14}$ Cf: Sherman N., The fabric of character. Aristotle's theory of virtue, Oxford University Press, Oxford1989.

${ }^{15}$ Cf: MANes F.,- NiRo M., Usar el cerebro. Conocer nuestra mente para vivir mejor. Planeta, Buenos Aires, 2014, 234-270.
} 
decisiones. La evolución del área frontal del cerebro está íntimamente relacionada con la aparición de moralidad humana. Es un área íntimamente relacionada con las estructuras emocionales ${ }^{16}$, tales como el sistema límbico a través de la amígdala, con el hipotálamo y con la sustancia gris periacueductal (Hardi y Holmes 1988) ${ }^{17}$. También están implicadas áreas como la corteza prefrontal dorsolateral y dorsomedial aunque estas son áreas más cognitivas que emocionales y están también involucradas en el procesamiento de la memoria operativa, la planificación y la atención. Estas áreas interactúan en el proceso normal de toma de decisiones. El haber puesto el acento en el control del comportamiento, en el análisis y las consecuencias ha contribuido a la falsa idea de que nos regimos solo por la racionalidad. Cuando está involucrada la vida propia y la de otras personas, no es fácil permanecer neutral desde el punto de vista emocional, ya que implican poder, sumisión, beneficios, valoraciones, etc. ${ }^{18}$. «Durante muchos años creímos ser "seres racionales (córtex) con sentimientos (límbico)", Hoy, los científicos acuerdan que el interruptor central del cerebro es nuestra parte emocional. Somos seres emocionales que aprendimos a pensar, y no máquinas pensantes que sentimos» ${ }^{19}$.

Estructuras cerebrales como el nucleo accumbens, tienen el papel de transferir información motivacional relevante en orden a reconocer situaciones que exijan conductas adaptativas ${ }^{20}$.

16 «El estado de ánimo influye muchísimo en esa capacidad. Generalmente los pacientes neuropsiquiátricos desarrollan patrones deficientes en la toma de decisiones coherentes con las manifestaciones clínicas y neuropsicológicas de la enfermedad. Por ejemplo, en situaciones típicas de juego, los depresivos demoran más tiempo en tomar decisiones $\mathrm{y}$, además, tienden a apostar menos que los grupos de control en situaciones favorables; los obsesivos prefieren la recompensa inmediata y no desarrollan una buena estrategia; los impulsivos apuestan enseguida (no esperan para analizar todas las posibilidades detalladamente); los extrovertidos, maníacos o desinhibidos se sienten más positivos, actúan decisivamente hacia potenciales de recompensas y apuestan muchos sin recolectar previamente toda la información necesaria. En diversas ocasiones es difícil saber si esto se debe a una genuina actitud de búsqueda de riesgo o consecuencia de la impulsividad que padecen». En MANES F.,- NiRO M., Usar el cerebro. Conocer nuestra mente para vivir mejor. Planeta, Buenos Aires, 2014, 235.

${ }^{17}$ Cf: Neurobiología y plasticidad neuronal, Material del Curso, Asociación EDUCAR, Buenos Aires 2014.

${ }^{18}$ Sin embargo hoy han reaparecido autores que postulan la vuelta a la concepción racionalista en cuanto al origen de nuestros juicios morales. Sam Harris es partidario del racionalismo moral y considera que la ciencia empírica ayudará a determinar las opiniones morales. En http://www.terceracultura.net/tc/? $\mathrm{p}=6879$ (Consultado el 27.5.14).

${ }^{19}$ BACHRACH E., Ágil mente. Aprende como funciona tu cerebro para potenciar tu creatividad y vivir mejor, Sudamericana, Buenos Aires 2013, 84. «Esto tiene lógica si pensamos que el límbico lleva más de doscientos millones de años sobre la Tierra y el córtex apenas cien mil años. La emoción tiene más dominio sobre nuestra razón. Por eso, la mayoría de las decisiones que tomamos en la vida no son concientes; la gran mayoría de ellas está dominada por una gran ráfaga de emociones (algunas liberadas de nuestras memorias, otras por emociones nuevas). Muchas veces nuestro consciente racional justifica decisiones que ya habíamos tomado antes de ser conscientes de ellas. En definitiva, el botón cerebral para comportarnos frente a las variadas situaciones cotidianas estás más influido por nuestras emociones que por la razón» Ibid 87.

20 «Hoy sabemos que la amígdala tiene más influencia en el córtex que éste sobre la amígdala. Por eso en ciertos momentos, las emociones dominan y controlan el pensamiento. Si estudiamos a los mamíferos, los surcos neuronales que van de la amígdala al córtex son muchos más fuertes que aquellos que van del córtex a la amígdala. Sabemos que los pensamientos pueden disparar con facilidad ciertas emociones, y lo hacen a través de la activación-adivinaron-de la amígdala. Pero también sabemos que no somos efectivos para apagar las emociones con los pensamientos, es decir, desactivar la amígdala» en BACHRACH E., Ágil mente. Aprende como funciona tu cerebro para potenciar tu creatividad y vivir mejor, Sudamericana, Buenos Aires 2013, 284-285. Las características de las vías que van de una 
Otro de los grandes aportes de la neurociencia actual es la superación de un dogma que hasta hace poco existía, por el cual se creía que el sistema nervioso era una estructura rígida e inmodificable ${ }^{21}$. El cerebro se transforma de manera constante a lo largo de toda nuestra vida. La experiencia y el ambiente tienen un protagonismo fundamental. El cerebro es un órgano adaptativo.

"Se denomina neuroplasticidad a la capacidad del sistema nervioso para modificarse y adaptarse a los cambios. Este mecanismo permite a las neuronas reorganizarse al formar nuevas conexiones y ajustar sus actividades en respuesta a nuevas situaciones o cambios del entorno"22.

Antes se postulaba que se nacía con una cantidad predeterminada de neuronas y éstas se conectaban entre sí de una manera para siempre. El cerebro no solo tiene la capacidad de modificarse incluso en la edad adulta, sino que también existe la producción de nuevas neuronas en algunas regiones del cerebro adulto. Uno de los lugares claves de la neuroplasticidad es a nivel de la conexión entre las neuronas. Cada vez que la memoria retiene información se generan nuevas sinapsis, se fortalecen otras, algunas se debilitan y otras se podan. Esta es la base neurobiológica de la formación de hábitos conductuales. Los hábitos ya adquiridos se pueden cambiar y los nuevos se pueden fortalecer. De manera tal que los circuitos cerebrales, generados por las nuevas prácticas, releguen los circuitos antiguos generados por los viejos hábitos hasta llegar a casi inactivarlos.

\section{Y todo ésto.... ¿qué significa?}

\section{Inclusión del dato neurobiológico en Filosofía y Teología Moral}

Los resultados de los estudios neurocientíficos, que muestran la activación de los centros emotivos, a la hora de decidir y de los racionales a la hora de justificar esa decisión, ofrecen a la ética Filosófica y Teológica elementos para una posterior reflexión.

Pero primero debemos preguntarnos, cómo se integra el dato neurobiológico en la reflexión tanto de la Filosofía como de la Teología. Con qué estatuto incorporamos el dato neurocientífico del comportamiento ético ${ }^{23}$. Actualmente contamos con una serie de autores, que se ubican en diferentes posiciones. En la relación entre filosofía y dato neurocientífico, existen básicamente dos. La primera consistiría en incorporar el dato

\footnotetext{
estructura a la otra y la activación de la amígdala en el stress son elementos significativos y que deben ser considerados a la hora de pensar las decisiones morales en la urgencia. Es importante saber que la activación por un peligro es mucho más duradera, rápida y difícil de apagar que la que se desencadena de una recompensa o un placer. Ibid. 268. Si almacenamos datos de posibles situaciones pensadas con anterioridad, tenemos más probabilidades de ser creativos en la urgencia. Ibid, 151-157. Para reaccionar creativamente es muy importante relajarse. Esto no solo beneficia en la reducción del estrés sino que baja el nivel de reactividad emocional y estimula los niveles de sincronicidad de las regiones no especializadas del cerebro, permitiendo más espacio en la mente para general ideas creativas o revelaciones que vengan de nuestro inconsciente. Ibid, 148-149.

${ }^{21}$ Cf.: MANES F.- NiRo M., Usar el cerebro. Conocer nuestra mente para vivir mejor. Planeta, Buenos Aires, 2014, 86-88.

${ }^{22} \mathrm{Ibid}, 86$.

${ }^{23}$ Cf.: CORTINA A., Neuroética, ¿moral como estructura o como contenido?, Conferencia dictada $1^{\circ}$ de septiembre de 2010 en el marco del III $^{\circ}$ Congreso Internacional Xavier Zubiri, Pontificia Universidad Católica de Valparaíso, Chile, en http://www.youtube.com/watch?v=QzM7 dKGMSs . (Consultado el 12.9. 2013).
} 
biológico como determinante del comportamiento ético y fundamentar a partir de allí una ética universal empíricamente demostrable de base cerebral, que sustituiría las éticas filosóficas y teológicas hasta ahora elaboradas. La segunda posición pregonaría aprovechar estos datos para entender la forma que tenemos los seres humanos de actuar éticamente y, a partir de allí, confrontar, enriquecer e iluminar nuestras teorías éticas y sentar las bases en orden a formar conciencias capaces de tomar decisiones éticas que nos vayan construyendo como mejores personas. Tanto la Psicología Moral, como la Filosofía y la Teología Moral se verían enriquecidas por el dato neurobiológico ya que nos pone en contacto con elementos constitutivos del sujeto que actúa. No se debe olvidar que las respuestas captadas por los estudios neurocientíficos son el resultado de un cerebro en evolución adaptativa. Y no podemos entender la adaptación como una instancia cerrada (mecanismos fijos de adaptación al medio para sobrevivir), sino que nos adaptamos adaptando al medio para poder realizar nuestros proyectos, de manera tal que la adaptación se entiende como una instancia de libertad, proyección, futuro, esperanza, creación, inteligencia, y posibilidad de aprovechar los medios para poner nuestros proyectos en obra ${ }^{24}$. Nos incardinamos en esta segunda propuesta.

\section{Formar para decidir}

El dato neurobiológico afirma la importancia preeminente de las emociones y la desafiante cualidad de la neuroplasticidad. Una formación que no parta de esta premisa, estará construyendo sobre datos no reales. El rol de las emociones es más importante de lo que se creía. Y este dato neurocientífico ilumina en dos dimensiones. Por un lado, nos hace entrar en el terreno de la formación de la conciencia con una dosis considerable de certeza en cuanto a hacia dónde deberíamos apuntar. Y por otro lado ilumina acerca del cómo hacerlo y con qué método. Las emociones pueden cultivarse y educarse, y allí debe focalizarse el mayor esfuerzo. No podemos esperar del dato neurobiológico la fundación de una nueva moral fundamental universal y concreta. De la estructura cerebral no podemos sacar un deber moral. Estaríamos cayendo nuevamente en una falacia naturalista que del " $E S$ " pretende derivar el " $D E B E$ ". Pero sí nos queda claro que este dato explicita de dónde partimos en nuestras conductas decisionales, a qué tendemos y cómo podemos crecer en orden a delinear libre y plenamente decisiones cada vez más humanas y humanizantes.

Educar emociones es también educar en el equilibrio. La emoción debe ser incorporada en forma sana y con elementos que ayuden a su integración y manejo. Eso es imposible de hacer sin una reflexión acerca del modelo antropológico de base y de los fines de la tarea para la cual se quiere entrenar. Es importante remarcar que la misma emoción tiene una valencia cognitiva importante, convirtiéndose, cuando es bien integrada, en una vía de abordaje de la realidad humana, enriqueciendo de algún modo al modelo antropológico del cual parte y al accionar profesional que se quiere lograr.

La neurociencia ha mostrado cómo las redes neuronales delinean nuestras conductas personales, especialmente en el ámbito de los hábitos. Estos se determinan como procesos sinápticos organizados en circuitos cerebrales que se van armando $y$ robusteciendo a fuerza de práctica conductuales ${ }^{25}$ Es evidente la convivencia de viejos

\footnotetext{
${ }^{24}$ Cf: Ibid.

25 «Cuando en el cerebro se enciende el sistema de comportamiento-recompensa, se libera una fuerte dosis de dopamina que nos motiva a volver a intentarlo y repetirlo. Durante algún tiempo este sistema de comportamiento repetitivo enciende el circuito de recompensa y otros sistemas neurológicos que nos motivan a mejorar la performance y hacerlo cada vez más fácil y con frecuencia» en BACHRACH E., Ágil mente. Aprende como funciona tu cerebro para potenciar tu creatividad y vivir mejor, Sudamericana,
} 
y nuevos circuitos y la posibilidad de debilitar y reforzar los que por el ejercicio de nuestra libertad y constancia elijamos afianzar. No solo debemos integrar el componente emotivo en la determinación de estos circuitos y en la formación de la conciencia para los juicios morales sino que urge una apuesta a la neuroplasticidad en la formación moral a lo largo de toda nuestra vida. En términos evangélicos podríamos decir: "¿Cómo puede uno nacer siendo ya viejo? ¿Puede acaso entrar en el seno de su madre y nacer?" (Jn 3, 4b).

La Filosofía Ética y la Teología Moral, especialmente desde la base de una Antropología cristiana, se enfrentan hoy con el gran desafío de integrar el dato neurobiológico y devolver al ser humano de hoy la posibilidad de una propuesta práctica y concreta de realización plena y del ejercicio de la propia libertad.

Buenos Aires 2013, 99. «Tenés que aprender a tocar un instrumento. Luego, practicá, practicá, practicá. Y finalmente, cuando estés arriba del escenario, olvídate de todo eso y déjalo gemir» Charlie Parker. 
BIBLIOGRAFIA

LIBROS

Abignente D., BAStianel S., Sulla formazione morale. Soggetti e itinerari, Il pozzo di giacobe, Trappani 2013.

BoIs M., Psychologie et Morale, Réception de la Psychologie cognitive-développementale au sein de la théologie morale post conciliaire, Thése doctorale, Universidad Pontificia Comillas, Madrid 2006.

CORSI M., Aproximaciones de la neurociencia a la conducta, Editorial El Manual Moderno, Guadalajara, México 2004.

Cortina, A., Neurofilosofía Práctica, Comares, Madrid 2012.

DAMASIO A., Y el cerebro creó al hombre. ¿Cómo pudo el cerebro generar emociones, sentimientos, ideas y el yo?, Destino, Barcelona 2012.

DISPENZA, J., Desarrolle su cerebro, Kier, Buenos Aires 2013.

GaZZANIGA M., Cuestiones de la mente. Cómo interactúan la mente y el cerebro para crear nuestra vida conciente, Herder, Barcelona 1998.

The ethical Brain, The science of our moral dilemmas, Danna Press, New

York 2005.

¿Qué nos hace humanos? La explicación cientifica de nuestra singularidad como especie. Paidós Ibérica, Barcelona 2010.

Quién manda aquí? El libre albeldrío y la ciencia del cerebro, Paidós

Ibérica, Barcelona 2012.

HAIDT J., The Happiness Hypothesis: Finding Modern Truth in Ancient Wisdom. Arrow books, London 2006.

HAUSER, M., Moral Minds. How Nature Designed Our Universal Sense of Right and Wrong, Brown Book Group Limited, Little 2007. , Mentes salvajes. ¿Qué piensan los animales?, Granica, Barcelona 2002.

KeENAN J., Virtudes de un cristiano, Mensajero, Bilbao 1999.

Lopez Frías F., y Alt (EDS.), Bioética, neuroética, libertad y justicia, Comares, Granada 2013.

Majorano S., La coscienza: Per una lettura cristiana, San Paolo, Milano 1994.

NUfFIELD COUNCIL ON BIOETHICS, Novel Technologies: interverning in the brain, ESP Colour Ltd, London 2013. 
Purves, D., et Alt., (Eds.), Neurociencia, Editorial Médica Panamericana, Madrid $2007^{30}$.

SAntoyo Velazco C., y otros, Proceso piscológico de la negociación y la toma de decisiones. Facultad de filosofía de la Universidad de México. 2001, México.

SHERMAN N., The fabric of character. Aristotle's theory of virtue, Oxford University press, 1989.

\section{CAPÍtULOS DE LIBROS COLECTIVOS}

CAstro CAmpolongo, C., "Reformular las convicciones. Un materialismo bien recibido en teología para pensar la teología de la creación en clave evolutiva», en FLORIO L., (ED.), Evolución y cristianismo. Un diálogo posible. Editorial Dunken, Buenos Aires 2007, 79-115.

ReHRAUER S., "Lo sviluppo morale del bambino», en Stefano Zamboni (Ed.), Etica dell'infanzia, Questioni aperte, Lateran University Press-Editiones Academiae Anfonsianae, Roma 2013.

\section{ARTíCULOS DE REVISTAS}

Boıs M., «Hacia una comprensión psico-teológico-moral del juicio moral», Moralia 31 (2008) 39-64.

CORTINA, A., "Ética del discurso ¿un marco filosófico para la neuroética?», Revista de Filosofía Moral y Política, N.o 48, enero-junio, 2013, 127-148.

Fumagalli M., AND PRIORI, A. ., "Funtional and clinical neuroanatomy of morality», Brain 18; 14(4), (2012),355-65.

Graham. J. \& HAIDT, J., «Beyond beliefs: Religión Bindsindividuals into Moral Communittes», Personality and Social Psychology Review 14, (2010), 140-150.

HAIDT J., «The emocional dog and Its Rational Tail: a Social Intuitionist Approach to Moral Judgment», Psychological Review, 4(2001)108,814-834.

MoyA, J., «Avances en Neurología e implicaciones éticas», Moralia 31 (2008) 97-119. , "Las emociones y la toma de decisiones morales», Moralia, 35, (2012),

155.177 «Problemas éticos en torno a la mejora del cerebro», 140 (2013) 26, 427448.

PÉREZ ZAFRILLA, P., «Implicaciones normativas de la psicología moral: Jonathan Haidt y El desconcierto moral», Daimon, 59 (2013),9-25.

CUNEO M.M., «Decisiones, emociones y bioética. Apuntes neurocientíficos» en Moralia 38, (2015) 179-191. 